\title{
A INFLUÊNCIA DO MERCADO DE TRABALHO E DO BACKGROUND FAMILIAR PARA O AVANÇO ENTRE NÍVEIS ESCOLARES NO BRASIL
}

\section{THE ROLE OF THE LABOR MARKET AND THE FAMILY BACKGROUND IN GETTING MORE EDUCATION IN BRAZIL}

Lindomar Pegorini Daniel ${ }^{1}$

Resumo: Sendo a educação apontada como eficiente mecanismo de transferência e redistribuição de renda, o presente artigo analisou como o background familiar e o mercado de trabalho impactam sobre a decisão de progressão entre níveis escolares no Brasil. O procedimento consiste em um stepwise para verificar se a ausência de certos fatores causa viés de omissão. Os resultados revelaram que a exclusão de características do background familiar causa viés de omissão de variável relevante, indicando que as mesmas são importantes na tomada de decisão quanto ao nível educacional. Fatores ligados ao mercado de trabalho e geográficos também se mostraram importantes, principalmente o fato de residir na zona rural, que impõe custos de transação adicionais para obter níveis mais elevados de educação. Constatouse que vários fatores estavam ligados direta ou indiretamente com a renda, evidenciando que esse seria um canal condicionante para o aumento da escolaridade (capital humano). Políticas públicas que foquem a mitigação da pobreza podem exercer efeitos positivos sobre a quantidade de educação no país. Por fim, chegou-se à conclusão de que mesmo significativos, as características consideradas exercem pouca influência sobre a decisão de completar níveis elevados de educação, que estaria mais ligada à habilidade inata do indivíduo.

Palavras Chave: Ciclos escolares; logit ordenado.

Abstract: Since education is an efficient mechanism for income transfer and redistribution, this paper examined the impact of family background and labor market on the decision of progression between levels of education in Brazil. The procedure consists in a stepwise to verify if the absence of certain factors cause omission bias. The results revealed that the exclusion of characteristics of family background cause bias omission of relevant variable, indicating that they are important in decision making regarding educational level. Geographical and labor market factors were also important, especially the fact of living in rural areas that impose additional transaction costs to obtain higher levels of education. We found that several factors are directly or indirectly with income, indicating that this channel would be a constraint to increased education (human capital). Public policies that focus on poverty reduction can exert positive effects on the amount of education. Finally, we find that even significant characteristics considered have little influence on the decision to complete higher levels of education, which would be more related to individual's innate ability.

Keywords: Education level; ordered logit.

\footnotetext{
${ }^{1}$ Professor Adjunto da Universidade do Estado de Mato Grosso. Doutor em Economia Aplicada pela Universidade Federal de Viçosa. E-mail: lindomar.pegorini@unemat-net.br
} 


\author{
JEL: I20; C25.
}

\title{
1 Introdução
}

Nesse artigo, aborda-se a questão do avanço entre ciclos escolares no Brasil. Atualmente a educação é considerada como fator chave para o aumento do bem estar social. Seus papéis como condicionador do aumento do capital humano, que leva ao crescimento e desenvolvimento econômico, redutor da desigualdade na distribuição de renda e da consequente redução da pobreza têm sido destacados tanto na literatura como na formulação de políticas públicas no Brasil e no mundo.

Segundo Hanushek et al (2001), na presença de externalidades, o investimento em educação como meio de redistribuição de renda leva a resultados Pareto superiores quando comparados a outros mecanismos de transferência de renda como o subsídio em impostos ou em salários (caso do Bolsa Família). Ainda, segundo Hanushek e Woessmann (2009), fatos estilizados como o fraco desempenho nas últimas décadas em termos de crescimento e desenvolvimento econômico da América Latina estariam ligados aos baixos níveis de educação e sua qualidade questionável.

Outro fato consolidado, desde Becker (1962) e Mincer (1974), é de que o investimento em educação resulta em retornos futuros em termos de maior produtividade e maiores ganhos de renda para os indivíduos. Apesar disso, Leon e Menezes-Filho (2002) argumentam que no Brasil o progresso educacional não parece responder a esses incentivos, como sugere o fato de que apenas 33\% da população com idade superior a 30 anos possuía nível médio ou superior em 2009. Por outro lado, a população nessa mesma faixa etária que não possuía 0 ensino fundamental completo estava em torno de 54\%.

Frente ao alto retorno econômico da educação e, consequentemente, sendo a mesma eficiente mecanismo de redistribuição de renda e superação da pobreza é de interesse dos policymakers conhecer os fatores que influenciam a escolha individual do avanço entre ciclos escolares. No entanto, observa-se que os estudos realizados se concentram sobre o retorno da educação e, atualmente, sobre a heterogeneidade desse retorno. Por outro lado, existem poucos trabalhos que tratam do porque as pessoas escolhem diferentes níveis de educação ou qual é o impacto dessa decisão para suas carreiras (ALTONJI; BLOM; MEGHIR, 2012).

No Brasil, o fato de existirem poucas pesquisas sobre o avanço entre ciclos escolares é surpreendente, devido à importância que o acesso ao ensino em todos os níveis recebe na definição de políticas públicas de educação. Segundo o IPEA, (2011), o país conta com serviços públicos estruturados de alfabetização, educação básica, educação superior e pós-graduação. O número de escolas disponíveis no sistema chega próximo a 180 mil. Além disso, na educação básica estão empregados cerca de 2 milhões de professores, dos quais 1,6 milhão na rede pública. No ensino superior, são quase 340 mil docentes, 120 mil em instituições públicas. 
Acompanhando a estrutura física e humana, ainda se verificam a distribuição de alimentos e refeições, livros e materiais didáticos, serviços de transporte escolar e o acesso aos meios digitais de aprendizagem e à internet para alunos da rede pública da educação básica. Atualmente, todo esse aparato custa por ano cerca de 5\% do Produto Interno Bruto (PIB), ou 161,2 bilhões de reais em 2009. De acordo com o novo Plano Nacional de Educação (PNE, 20112020), esse gasto deve alcançar, até 2020, 7\% do PIB, uma das 20 metas propostas, que contemplam ainda a universalização e o aumento da qualidade do ensino em todos os níveis.

Nesse sentido, dada a ênfase no acesso ao ensino (formação de capital humano) dois pontos podem ser notados quando as pessoas decidem não aumentar seu nível de escolaridade: vagas ficam ociosas e o nível de capital humano não cresce no montante esperado. Isso representa um problema econômico na medida em que recursos públicos escassos são desperdiçados, deixam de ser aplicados em outras áreas, e o efeito multiplicador da educação fica aquém de seu potencial.

Mesmo pequenas perdas de eficiência podem resultar em perda expressiva de recursos, além de outros custos humanos, (LEVIN, 1989). Por isso, alguns mecanismos têm sido implementados pelas instituições públicas visando melhor alocação dos recursos educacionais, como o aumento da taxa de ocupação das vagas nas universidades por meio do Sistema de Seleção Unificada (Sisu), dentre outros.

Portanto, este estudo tem como objetivo identificar os fatores relevantes para determinar o avanço entre ciclos escolares no Brasil, em especial, verificar a importância das características familiares e do mercado de trabalho para a decisão quanto ao nível de graduação educacional. Os resultados são importantes para a formulação de políticas e ações de cunho público a fim de aumentar a eficiência da alocação de recursos e o nível de capital humano, reduzindo, consequentemente, a concentração existente nos níveis de renda e gerando ganhos de bem estar social.

O artigo encontra-se organizado em mais 4 seções além desta introdução. A seção 2 trata do modelo teórico utilizado para explicar a decisão do indivíduo para o avanço entre ciclos escolares. Na seção 3, apresenta-se a metodologia aplicada para alcançar o objetivo proposto, elucidando o método estatístico empregado e a seleção de variáveis. O procedimento consiste na estimação de uma equação de escolhas ordinais, conhecida como logit ordenado. A estimação foi operacionalizada pelo método de Pseudo Máxima Verossimilhança (MPV), utilizando como amostra os microdados da Pesquisa Nacional por Amostra de Domicílio (PNAD) 2009. A seção 4 apresenta os resultados e a discussão do trabalho, observando as características relevantes para o avanço entre ciclos escolares. Por fim, a seção 6 apresenta as considerações finais.

\section{Modelo teórico}

Esta seção descreve os fundamentos teóricos que sustentam a decisão de avanço entre ciclos escolares de um agente representativo maximizador de utilidade. As pessoas tomam decisões durante seu ciclo de vida. Elas fazem a escolha entre aumentar ou não seu nível de escolaridade baseados na sua função de utilidade, essas são informações relevantes que devem ser levadas em consideração na formulação de políticas públicas. Um indivíduo possui uma 
função de utilidade que expressa suas preferências entre avançar de ciclo escolar (continuar estudando), ou consumir (desenvolver outra atividade, trabalhar).

A função de utilidade do indivíduo i pode ser denotada como:

$$
u_{c}^{i}\left(C_{c}^{i} \mid a^{i}, \theta^{i}, \Omega^{\mathrm{i}}, V^{\mathrm{i}}, \varepsilon_{c}^{i}\right), \quad \text { onde } i=1, \ldots, n
$$

onde $c$ denota o ciclo escolar ou a escolaridade do indivíduo (fundamental incompleto, fundamental, médio, superior, pós graduação). A utilidade do indivíduo $i$ depende de seu nível de consumo $C_{c}^{i}$, e de um choque aleatório de preferências $\varepsilon_{c}^{i}$. Além disso, a utilidade é função da habilidade inata $a^{i}$, de um vetor de características pessoais $\theta^{i}$, de um conjunto de características da família $\Omega^{\mathrm{i}}$ e de um vetor de características do mercado de trabalho $V^{\mathrm{i}}$ (ALTONJI, BLOM e MEGHIR, 2012).

A decisão entre completar um novo ciclo escolar ou não depende de algumas condições. Primeiro, a disposição em cursar um novo ciclo escolar pelo indivíduo depende da utilidade atribuída pelo mesmo às suas necessidades de consumo, caso confira demasiado grau de utilidade para o consumo ele se dedicaria integralmente ao objetivo de suprir suas demandas, que estaria adicionalmente ligada à restrição orçamentária do indivíduo, ou seja, a educação não seria sua prioridade. Segundo, essa decisão é função das características pessoais do agente e de suas habilidades inatas, ou seja, do custo de oportunidade entre trabalhar ou desenvolver outras atividades. Terceiro, a influência dos pais e as condições familiares do indivíduo são determinantes para a tomada de decisão. Por fim, as condições do mercado de trabalho e do local de residência também são condicionantes dessa decisão.

Para que o indivíduo decida completar um determinado nível de educação ele deve ter completado o ciclo anterior, sendo esta uma condição necessária, mas não suficiente para avanço entre ciclos escolares. Integrando a primeira condição, a utilidade de um nível de ensino adicional deve ser maior que a atual, dessa forma:

$$
u_{2}^{i}\left(C_{2}^{i} \mid a^{i}, \theta^{i}, \Omega^{\mathrm{i}}, \varepsilon_{2}^{i}\right)>u_{1}^{i}\left(C_{1}^{i} \mid a^{i}, \theta^{i}, \Omega^{\mathrm{i}}, \varepsilon_{1}^{i}\right), \quad \text { onde } i=1, \ldots, n
$$

Um problema inerente a este modelo é que a utilidade do indivíduo é uma variável latente, não observável. Não é possível conhecer a utilidade dos agentes, apenas se a decisão de elevar o nível de ensino foi tomada ou não. Nesse caso, o problema de interesse é saber qual a probabilidade do indivíduo avançar de um ciclo para outro, dadas as condições conhecidas sobre o mesmo. Nesse contexto, a regra de decisão de avanço entre níveis de escolaridade será: o indivíduo completa um novo ciclo de educação caso sua utilidade supere sua utilidade de reserva.

Para a análise do avanço entre ciclos escolares no Brasil, utiliza-se o arcabouço teórico do capital humano, baseado em Becker (1962). Essa abordagem parte do princípio de que o treinamento e a experiência são capazes 
de desenvolver as habilidades individuais durante o período de vida. Postula ainda, que os rendimentos individuais são função dessas habilidades desenvolvidas e incorporadas ao longo do tempo, ou seja, rendimentos maiores estariam associados a um nível maior de acumulação dessas habilidades, do estoque de capital humano.

\section{Metodologia}

\subsection{O modelo de escolhas ordinais}

Para atender ao objetivo proposto pelo trabalho, verificar a influência que o background familiar e o mercado de trabalho exercem sobre a decisão de continuar estudando, é necessário levar em consideração o ordenamento natural envolvido nesta decisão e os desafios e limitações existentes na análise empírica desse tipo de problema.

Nesse caso a variável de interesse, nível de escolaridade, possui uma ordem intrínseca. O indivíduo deve, necessariamente, concluir um ciclo escolar para então iniciar nível subsequente. A existência de hierarquização natural nos níveis escolares é de extrema importância para a definição do modelo empírico de análise como enfatizam Long e Freese (2006), pois o fato da decisão ou resposta apresentar a possibilidade de ordenamento não significa que ela deva ser analisada dessa forma.

Uma ferramenta útil nesse tipo de problema são os modelos de variável dependente qualitativa ordinal. Pode-se denotar o problema inerente com um modelo de variável latente:

$$
u_{i}^{*}=\mathbf{x}_{\mathrm{i}}^{\prime} \beta+\varepsilon_{i}
$$

onde $u_{i}^{*}$ é a variável latente não observável (utilidade), $\mathbf{x}$ é um vetor que reúne as características pessoais e familiares dos indivíduos, além dos fatores do mercado de trabalho $\left(a^{i}, \theta^{i}, \Omega^{\mathrm{i}}, V^{\mathrm{i}}\right)$ e $\varepsilon_{i}$ é o termo de erro com as propriedades desejáveis. $O$ conjunto de parâmetros $\beta$ reflete a influência das características supracitadas sobre a utilidade do indivíduo.

Em essência a decisão é modelada sob a pressuposição de que um conjunto de variáveis exógenas determina uma variável endógena não observável (latente), $u^{*}$, utilidade neste caso. Se a utilidade excede certo limiar, o indivíduo toma a decisão de cursar mais um nível escolar, caso contrário a decisão é negativa. Como se observa somente o avanço ou não entre ciclos escolares tem-se:

$$
\left(u_{i}=j\right) \text { se }\left(\alpha_{j-1}<u_{i}^{*} \leq \alpha_{j}\right)
$$

onde os valores $\alpha^{\prime} s$ representam os limites, limiares ou thresholds para mudança de decisão entre avançar ou não entre determinado ciclo escolar. Segundo Cameron e Trivedi (2005) a probabilidade associada ao indivíduo decidir entre um ciclo escolar e outro pode ser representada como: 


$$
\operatorname{Prob}\left(Y_{i}=j \mid \mathbf{x}\right)=F\left(\alpha_{\mathrm{j}}-\mathbf{x}_{\mathrm{i}}^{\prime} \beta\right)-F\left(\alpha_{\mathrm{j}-1}-\mathbf{x}_{\mathrm{i}}^{\prime} \beta\right)
$$

onde $F$ é a distribuição de probabilidade contínua de $\varepsilon_{i}$.

O conjunto de parâmetros $\beta$ reflete o impacto na probabilidade causado por mudanças no vetor $\mathbf{x}$, ou seja, os sinais dos parâmetros determinam se a variável latente aumenta ou decresce com a variação dos fatores analisados.

Em princípio, qualquer distribuição de probabilidade contínua seria suficiente para solucionar o problema. Porém, devido a sua conveniência matemática, neste artigo, utilizou-se a distribuição logística:

$$
F\left(u_{i} \mid \mathbf{x}\right)=\frac{e^{\mathbf{x}^{\prime} \beta}}{1+e^{\mathbf{x}^{\prime} \beta}}=\Lambda\left(\mathbf{x}_{\mathbf{i}}^{\prime} \beta\right)
$$

A notação $\Lambda($.$) é usada para indicar a função de distribuição logística$ acumulada. Este modelo é conhecido como logit ordenado. Para o trabalho proposto, calculam-se as probabilidades das pessoas elevarem seu nível escolar, ou seja, quais fatores seriam importantes para o avanço entre ciclos escolares.

Pode-se, ainda, definir o efeito marginal de alguma característica pessoal ou familiar sobre a probabilidade de o indivíduo avançar determinado nível escolar:

$$
\frac{\partial \operatorname{Prob}\left(Y_{i}=j \mid \mathbf{x}\right)}{\partial \mathbf{x}_{\mathbf{i}}}=\left[\Lambda^{\prime}\left(\alpha_{\mathbf{j}}-\mathbf{x}_{\mathbf{i}}^{\prime} \beta\right)-\Lambda^{\prime}\left(\alpha_{\mathbf{j}-1}-\mathbf{x}_{\mathbf{i}}^{\prime} \beta\right)\right] \beta
$$

onde $\Lambda^{\prime}($.$) indica a derivada de \Lambda($.$) , em que o lado esquerdo da equação (7)$ representa o operador de diferencial da regressão do avanço entre ciclos escolares com relação ao determinante $i$, no lado direito, o primeiro termo representa a interação entre as probabilidades e o segundo termo o coeficiente da variável $i$. Como, em geral, o efeito marginal vai ser diferente para cada indivíduo, é comum calculá-lo no ponto médio da variável.

Outra peculiaridade apresentada pelo modelo de escolhas ordinais é a pressuposição de razões proporcionais, no caso da distribuição logística, que não permite a heterogeneidade individual entre as diferentes categorias. Nesse caso os coeficientes angulares serão os mesmos para os diferentes níveis escolares. Não se inclui intercepto na equação, em seu lugar encontram-se os limiares ou thresholds. Contudo o pressuposto não se mostra restritivo à presente análise, pois como citado anteriormente existe uma ordenação natural na variável de interesse que fundamenta o uso do modelo. No entanto, a literatura lista testes como o de Brant (1990) e de Wolfe e Gould (1998) para verificar a validade do pressuposto, porém os testes não suportam surveys com desenho amostral complexo como a Pesquisa Nacional por Amostra de Domicílio (PNAD) utilizada pelo presente estudo (LONG e FREESE, 2006).

\subsection{O modelo empírico}

A população considerada na análise é composta de indivíduos com idade acima de 10 anos. As variáveis utilizadas para explicar o avanço entre ciclos escolares concentram características pessoais e familiares (background 
familiar), do mercado de trabalho e geográficas e foram selecionadas de acordo com as evidências de Altonji, Blom e Meghir (2012), Souza, Ponczek e Oliva (2011), Corseuil, Santos e Foguel (2001), Carvalho et al. (2006), Farber e Gibbons (1996), Mincer (1974), Oaxaca (1973) e Becker (1962). Em resumo, os fatores considerados para o avanço entre ciclos escolares são:

$U_{i}{ }^{*}=\beta_{1}$ idade $_{i}+\beta_{2}$ branco $_{i}+\beta_{3 \text { masc }_{i}}+\beta_{4}$ pesref $_{i}+\beta_{5}$ transfrenda $_{i}+\beta_{6}$ renfampc $_{i}+$ $\beta_{7 n^{n}} \beta_{i}+\beta_{8}$ escpesref $i+\beta_{9}$ civilpesref $_{i}+\beta_{10}$ horastrabpesref $_{i}+\beta_{11}$ expesref $_{i}+$

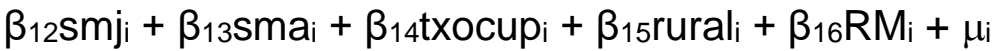

em que i representa o indivíduo; $\bigcup_{i}^{*}$, é a utilidade do indivíduo em aumentar seu nível de educação; $\beta_{j}(j=1$ a 16) são os parâmetros a serem estimados; idade, é a idade do indivíduo em anos; branco, é uma variável dummy que indica a raça, com o grupo base representando indivíduos que não declararam-se brancos; masc, é uma variável dummy que indica o gênero, com o grupo base representando o sexo feminino; pesref, variável categórica que aponta se o indivíduo é a pessoa de referência da família, com o grupo base representado os que não são responsáveis pela família; transfrenda, variável dummy que indica se o indivíduo aufere algum tipo de renda que não seja proveniente do trabalho como juros, pensões, previdência ou programas sociais, o grupo base refere-se aos indivíduos que não auferem renda que não seja do trabalho; renfampc, representa a renda familiar per capita; npes, é o número de residentes do domicílio; escpesref, escolaridade, em anos de estudo, da pessoa de referência da família; civilpesref, é uma variável dummy que indica se a pessoa de referência da família é casada, o grupo base refere-se às demais situações; horastrabpesref, representa o número de horas habitualmente trabalhadas pela pessoa de referência; expesref, é a experiência no mercado de trabalho da pessoa de referência; smj e sma, são o salário hora médio de indivíduos entre 14 e 20, e 26 e 65 anos de idade, respectivamente; txocup, representa a taxa de ocupação; rural, é uma variável binária que representa o local de residência do indivíduo, onde o grupo base refere-se à zona urbana; RM, como a anterior, no entanto refere-se à indivíduos que residem nas regiões metropolitanas; e $\mu$ é o termo de erro da equação.

O background familiar é formado pelas características individuais e familiares. Segundo Altonji, Blom e Meghir (2012), Cameron e Heckman (1998 e 2001), dentre outros, o background familiar é determinante para a decisão de avanço entre ciclos escolares. As características individuais incluídas foram idade, raça/cor, gênero, condição na unidade familiar e beneficiário de outros tipos de renda que não seja proveniente do trabalho.

A decisão entre estudar ou desenvolver outra atividade (trabalhar) ou exercer as duas de forma conjunta dependem, além de outros fatores, da habilidade inata do indivíduo. Nesse sentido, apesar de omitida, outras variáveis podem captar seu efeito (GRILICHES, 1977) e (FARBER; GIBBONS, 1996). Da variável idade, espera-se que quanto maior a idade do indivíduo menor seja a probabilidade de avanço entre os ciclos escolares. Isso se deve ao fato de que quanto maior a idade maior é a probabilidade de estar somente trabalhando, devido às maiores responsabilidades e à elevação do peso do trade off entre estudo e consumo.

A característica gênero foi inserida para controlar a heterogeneidade inerente às preferências de homens e mulheres quanto à decisão de elevação 
do nível de educação, mas também para captar possíveis efeitos de discriminação de gênero. A variável de raça/cor foi incluída por motivos similares.

Quanto ao fator pessoa de referência, este foi adicionado para controlar situações que exigem maior responsabilidade do indivíduo para com as necessidades de consumo próprias ou da família. Essa situação aumenta a demanda por trabalho que consequentemente demanda tempo e concorre com a decisão de continuar estudando.

A variável transferência de renda tem o objetivo de observar situações nas quais os agentes possuem fontes de renda alternativas que não provenientes do trabalho e qual seria a influência desse fato na decisão de completar novos ciclos de educação.

As características familiares incluem renda familiar per capita, número de pessoas no domicílio, escolaridade, estado civil, experiência e horas habitualmente trabalhadas por mês da pessoa de referência da família. Apesar de não representar diretamente a influência dos pais, supõe-se que a pessoa de referência, que muitas vezes coincide com a posição dos pais, seja determinante para as decisões dos indivíduos.

Para a renda familiar, escolaridade, experiência e estado civil da pessoa de referência espera-se uma relação direta, ou seja, quanto melhor a estrutura familiar maior a probabilidade do indivíduo alcançar maiores níveis de educação. Uma explicação para o fato é a de que quanto maior a renda familiar e a escolaridade da pessoa de referência maiores serão as condições da família e menor será a dependência da mesma para com o indivíduo, que pode continuar estudando (SOUZA; PONCZEK; OLIVA, 2011). Além disso, maior escolaridade e experiência dos pais, nesse caso da pessoa de referência, promovem incentivos aos filhos de modo a alcançar maiores níveis escolares, principalmente devido aos maiores níveis de produtividade e retorno futuro.

Em relação aos fatores número de pessoas no domicílio e horas trabalhadas pela pessoa de referência, acredita-se que exista relação inversa para com a decisão de estudar (CORSEUIL; SANTOS; FOGUEL, 2001). Maior número de pessoas no domicílio leva a maior razão de dependência e aumento do trade-off entre estudar e trabalhar. Quanto às horas trabalhadas, quanto mais tempo trabalhando menor é o tempo que a pessoa de referência dedica à sua família reduzindo sua influência sobre o avanço entre níveis de educação.

Quanto às características do mercado de trabalho, foram incluídas o salário médio local de jovens e adultos e a taxa de ocupação local. Os fatores de salário médio local tanto para jovens como para adultos foram construídos para todos os estados e regiões metropolitanas com o objetivo de controlar o tamanho do trade off entre estudar e consumir (trabalhar). Para ambas as variáveis, salário médio dos jovens e dos adultos, espera-se uma relação inversa com a probabilidade do indivíduo elevar sua escolaridade, dada a elevação do trade off que aumentos no salário médio representariam.

A taxa de ocupação foi incluída para captar a influência da probabilidade de emprego sobre o avanço entre ciclos escolares. Essa variável consiste na razão entre a população ocupada local sobre a população economicamente ativa local.

Variáveis de localidade de residência foram incluídas para avaliar a importância que fatores locais e custos de transação (transporte e deslocamento) exercem sobre a decisão de completar níveis de ensino. Foram inseridos fatores dicotômicos para controlar a heterogeneidade entre residir na 
zona urbana e rural, além de residir em regiões metropolitanas. Portanto esperase que a variável rural apresente sinal negativo, ou seja, se a pessoa reside na zona rural, a probabilidade de avanço entre ciclos escolares diminui, uma vez que, possuam em geral, maiores dificuldades de acesso ao local de estudo.

Para verificar a importância que características individuais, familiares, do mercado de trabalho e fatores locais possuem sobre a decisão de avanço entre ciclos escolares, adotou-se como estratégia empírica o procedimento conhecido como stepwise, ou seja, comparam-se os diferentes modelos com especificações distintas adicionando-se ao modelo base, de características individuais, as características familiares, do mercado de trabalho e do local de residência. A partir dessa comparação pode-se inferir se existe viés de omissão de fatores ligados à decisão de avanço entre ciclos escolares.

\section{Resultados e discussões}

\subsection{Análise descritiva}

A Tabela 1 apresenta estatísticas descritivas e definições das variáveis utilizadas. A variável categórica ordenada que indica o avanço entre ciclos escolares foi baseada na escolaridade dos indivíduos, e está dividida em seis categorias ou ciclos escolares: fundamental incompleto, que englobam notórios $37,1 \%$ da população brasileira, ou seja, que não completaram ou simplesmente não fizeram o ensino fundamental. Cursando o ensino fundamental, com 13,7\% da população. Fundamental completo que compreende $16,5 \%$ da população, médio completo que abrange cerca de $25,6 \%$ da população, superior completo com $6,8 \%$ e pós-graduação com $0,04 \%$ da população.

Tabela 1: Estatísticas descritivas das variáveis, 2009

\begin{tabular}{|c|c|c|c|}
\hline Variáveis & Média & $\begin{array}{l}\text { Desvio } \\
\text { padrão }\end{array}$ & Descrição \\
\hline \multicolumn{4}{|l|}{ Variável dependente } \\
\hline CICLO & & & Maior nível escolar completado pelo indivíduo (c) \\
\hline Fundamental incompleto & 0.371 & 0.002 & Se não possui nem cursa ensino fundamental (d) \\
\hline Cursando fudamental & 0.137 & 0.001 & Se está cursando ensino fundamental (d) \\
\hline Fundamental & 0.165 & 0.001 & Se possui ensino fundamental (d) \\
\hline Médio & 0.256 & 0.002 & Se possui ensino médio $(\mathrm{d})$ \\
\hline Superior & 0.068 & 0.001 & Se possui nível superior (d) \\
\hline Pós-graduação & 0.0041 & 0,001 & Se possui pós-graduação (d) \\
\hline \multicolumn{4}{|l|}{ Características individuais } \\
\hline Idade & 36.22 & 18.19 & Anos de idade do indivíduo \\
\hline Branco & 0.446 & 0.497 & Raça - 1 se branco $(d)$ \\
\hline Masculino & 0.481 & 0.500 & Gênero - 1 se masculino (d) \\
\hline Pessoa de referência & 0.385 & 0.487 & Condição na família - 1 se pessoa de referência (d) \\
\hline Transferência de renda & 0.050 & 0.218 & Recebe algum tipo de renda que não seja do trabalho (d) \\
\hline \multicolumn{4}{|l|}{ Características Familiares } \\
\hline Renda familiar per capita & 0.671 & 1.168 & Renda mensal em mil reais por pessoa da família \\
\hline Número de pessoas & 3.971 & 1.836 & Número de pessoas no domicílio \\
\hline Escolaridade da pessoa de referência & 7.127 & 4.647 & Anos de escolaridade da pessoa de referência \\
\hline Estado civil da pessoa de referência & 0.610 & 0.488 & Estado civil, pessoa de referência - 1 se casado (d) \\
\hline Horas trab. pela pessoa de ref. & 132.8 & 88.32 & Horas trabalhadas por mês pela pessoa de referência \\
\hline Experiência no trab. da pes. de ref. & 24.16 & 17.08 & Anos de experiência no trabalho da pessoa de referência \\
\hline
\end{tabular}




\section{Mercado de trabalho}

Salário médio local dos jovens

Salário médio local dos adultos

Taxa de ocupação

$\begin{array}{cc}2.49 & 1.34 \\ 4.58 & 1.36 \\ 91.23 & 2.68\end{array}$

$0.152 \quad 0.359$
Salário-hora médio local, pessoas entre 14 e 20 anos Salário-hora médio local, pessoas entre 26 e 65 anos

Probabilidade de emprego local

Características geográficas

Rural

$0.340 \quad 0.473$

Se reside na zona rural $(d)$

Nota: Desenho amostral considerado para estimação das médias populacionais. Variáveis indicadas com (c) são categóricas assumindo valores inteiros, indicadas com (d) são dicotômicas assumindo valor 1 se possui o atributo e 0 caso contrário. A população considerada é de 158,104,765 habitantes.

Pela análise da Tabela 1, constata-se que grande parcela da população ainda não possui ou não completou o ensino fundamental. $O$ baixo estoque de capital humano pode limitar de forma contundente o crescimento do país (HANUSHEK; WOESSMANN 2009). Entre as características que podem estar ligadas ao baixo nível de educação do Brasil destacam-se o baixo nível do background familiar médio.

O baixo nível médio de renda per capita $\mathrm{R} \$ 671,00$ mensais e o fato de apenas $5 \%$ da população receberem rendas adicionais que não sejam provenientes do trabalho demandam maior esforço dos indivíduos para suprir suas necessidades de consumo. Fato correlacionado é o do baixo nível médio de escolaridade da pessoa de referência da família, 7,1 anos. A taxa de ocupação média também sugere que o tempo para estudar sofre alta concorrência com o tempo destinado ao trabalho. Em suma, o baixo nível de escolaridade da população brasileira parece ser resultado de vários fatores, mas principalmente de baixo nível de background familiar e de renda.

\subsection{Background familiar, mercado de trabalho, local de residência e avanço} entre ciclos escolares

Apresenta-se a seguir, os resultados das estimações do logit ordenado para a decisão de avanço entre ciclos escolares. A Tabela 2 expõe as características pessoais, familiares, do mercado de trabalho e geográficas, os valores dos coeficientes e dos limiares ou thresholds, as previsões dos modelos e medidas de ajuste.

Tabela 2: Resultados para o modelo de avanço entre ciclos escolares, 2009

\begin{tabular}{lcccc}
\hline & \multicolumn{4}{c}{ Características } \\
\cline { 2 - 5 } & (i) Individuais & (ii) Familiares & $\begin{array}{c}\text { (iii) Mercado } \\
\text { de Trabalho }\end{array}$ & (iv) Geográficas \\
\hline Idade & -0.029 & -0.040 & -0.041 & -0.041 \\
Branco & 0.682 & 0.080 & 0.149 & 0.151 \\
Masculino & -0.220 & -0.248 & -0.249 & -0.247 \\
Pessoa de referência & 0.197 & 0.134 & 0.140 & 0.140 \\
Transferência de renda & 0.319 & $-0.023)$ & $-0.022)$ & $(-0.022)$ \\
Renda familiar per capita (In) & & 0.640 & 0.668 & 0.663 \\
Número de pessoas & & -0.027 & -0.029 & -0.029 \\
Escolaridade da pes. de ref. & & 0.259 & 0.258 & 0.257 \\
Estado civil da pes. de ref. & 0.159 & 0.166 & 0.171 \\
Horas trab. pela pes. de ref. & & -0.003 & -0.002 & -0.002 \\
Experiência da pes. de ref. & & 0.004 & 0.004 & 0.004 \\
Salário médio local dos jovens (In) & & & -0.417 & -0.456 \\
Salário médio local dos adultos (In) & & & $(0.067)$ & $0.049)$ \\
Taxa de ocupação & & & -0.041 & -0.029 \\
Rural & & & & -0.107 \\
Região metropolitana & & & & 0.097 \\
Previsão do modelo & & & & \\
Fundamental incompleto & & & & \\
\end{tabular}

Revista de Estudos Sociais | Faculdade de Economia - UFMT | Cuiabá - MT 
Cursando fundamental

Fundamental

Médio

Superior

Pós graduação

Teste F

Limite $\alpha 1$

Limite $\alpha 2$

Limite $\alpha 3$

Limite $\alpha 4$

Limite $\alpha 5$
0.141
0.156
0.242
0.067
0.0041
2360.57
-1.238
-0.622
0.086
1.948
4.897

Nota: A variável dependente é a variável categórica Ciclo Escolar. Todos os coeficientes são estatisticamente significantes ao nível de $1 \%$ exceto os que estão destacados entre parênteses, estes não foram significativos ao nível de $10 \%$. (In) indica a utilização do logaritmo natural na especificação do modelo.

Os coeficientes reportados indicam se a variável latente, utilidade de avanço entre ciclos escolares, aumenta ou decresce em função dos fatores condicionados. Praticamente todos os coeficientes foram significantes ao nível de $1 \%$. Por esta razão omitiram-se os erros padrão dos mesmos e identificou-se entre parênteses os que não foram significativos ao nível de $1 \%$.

O modelo (i) inclui apenas variáveis que controlam características pessoais. Quando ocorre o controle para características familiares, no modelo (ii), algumas características individuais alteram-se significativamente. $\mathrm{O}$ efeito dos fatores cor/raça e transferência de renda sofrem mudanças substancias, sugerindo que parte do impacto dessas variáveis devia-se à omissão das características da família. Dessa forma, conclui-se que características familiares são importantes na tomada de decisões quanto ao nível educacional, corroborando com os estudos de Altonji, Blom e Meghir (2012) e Souza, Ponczek e Oliva (2011).

No modelo (iii), ao inserirem-se fatores referentes ao mercado de trabalho local, ocorreram apenas mudanças pontuais nos coeficientes apesar dos fatores adicionais. Embora estatisticamente importantes para explicar a mudança na utilidade ligada ao avanço entre ciclos escolares, com exceção do salário médio entre 26 e 65 anos, não se observou viés relevante quanto à omissão dos fatores do mercado de trabalho.

Já a inserção das variáveis de controle para local de residência zona rural, tendo a zona urbana como base, e região metropolitana, tendo como base a não metropolitana, no modelo (iv), foram significativas, no entanto não implicaram em grandes mudanças em outras variáveis para explicar o avanço entre níveis escolares. Apesar de não resultarem em grandes alterações está claro que características geográficas como local de residência e custos de transação são importantes para essa decisão. Além disso, resultados semelhantes foram encontrados por Souza, Ponczek e Oliva (2011).

As demais informações contidas na Tabela 2 não permitem maior distinção entre os modelos. Os valores previstos pelos modelos são muitos próximos, além disso, os limites ou thresholds são significativos em quase todas as especificações exceto no modelo (iv), onde o limite a4 não o é, indicando que naquele caso as seis categorias de ciclos escolares poderiam colapsar em cinco. Nesse caso, concluiu-se que o modelo (iv) seria o mais adequado para a análise do avanço entre ciclos escolares uma vez que possui melhor especificação em relação aos outros. Portanto o modelo (iv) passará a ser usado como referência para a análise a partir de então.

Em relação ao impacto das variáveis sobre a variável latente utilidade, ligada ao avanço entre ciclos escolares, o modelo (iv) mostra que dentre as características individuais a idade e o fato de ser do gênero masculino diminuem 
a utilidade ou as chances de elevação do nível escolar, enquanto que raça/cor e ser a pessoa de referência da família possuem efeito contrário, aumentam a utilidade.

Com maior possibilidade de acesso ao ensino, as pessoas mais jovens têm associado à educação maior utilidade ou retorno futuro em termos de rendimento (MINCER, 1974). Além disso, o fato de estar acima da idade considerada habitual para determinado ciclo escolar diminui a utilidade do indivíduo em estudar devido ao crescente trade off existente entre maior nível de educação e consumo. Em termos práticos a escolaridade média se reduz de 7,8 para 6,4 anos quando se comparam a população abaixo e acima da idade média de 36 anos.

A mesma interpretação pode ser estendida para o gênero, as mulheres têm em média 0,4 anos a mais de estudo que os homens. Se muitas evidências apontam a discriminação de gênero como responsável pela discrepância salarial existente entre homens e mulheres, inclusive na mesma função, as mulheres têm atribuído maior utilidade ao avanço entre ciclos escolares para mitigar essa diferença de rendimentos, como aponta estudo de Pegorini, Castro e Lima (2014). Pelos mesmos motivos, tradição, cultura ou discriminação, a população branca possui em média 1,4 anos a mais de escolaridade que a não branca, ou seja, brancos têm maior probabilidade de obter níveis mais elevados de educação.

Ser a pessoa de referência da família, indubitavelmente, confere maior responsabilidade ao indivíduo em termos de necessidades de consumo da família ou domicílio. Dado o resultado, o indivíduo parece estar atribuindo maior utilidade ao consumo futuro dedicando-se mais a aumentar o nível de educação.

Por fim, se o indivíduo recebe algum tipo de transferência de renda, a possibilidade do mesmo aumentar seu nível de escolaridade não é relevante. Esse evento está associado à inclusão da renda familiar no modelo, como a transferência de renda faz parte da renda familiar esta capta toda a sua influência, que pode ser vista pelo modelo (i), onde receber algum tipo de renda que não seja proveniente do trabalho eleva a utilidade de estudar. Caso o indivíduo possa substituir trabalho por lazer ou por outra prioridade como a educação, e alcançar níveis superiores de satisfação no presente ou em seu horizonte futuro, posto que obtenha renda de outra fonte que não o trabalho, ele estará mais propenso em fazê-lo.

Para as características familiares, como esperado, verifica-se que a renda per capita familiar, escolaridade, estado civil e experiência no mercado de trabalho da pessoa de referência possuem relação direta com a utilidade de avanço entre ciclos escolares e número de pessoas no domicílio e horas trabalhadas reduzem a utilidade.

A estrutura familiar é importante para a tomada de decisão do indivíduo, quando a renda familiar e a educação dos pais, nesse caso da pessoa de referência, são elevadas as chances de o indivíduo cursar níveis mais elevados de ensino aumentam. Se a pessoa de referência é casada, a utilidade também aumenta, novamente, esse evento reforça a ideia de que a estrutura familiar tanto em relação à renda quanto à unidade familiar contribuem para que o avanço entre ciclos escolares seja uma decisão mais provável.

Ainda quanto aos eventos da escolaridade e experiência da pessoa de referência exercerem efeitos positivos sobre a educação do indivíduo, esses fatos são atribuídos à maior valorização que é conferida à educação, 
incentivando-o a elevar seu nível de escolaridade e, mesmo, dando suporte financeiro. Já com relação ao número de pessoas no domicílio e maior número de horas trabalhadas pela pessoa de referência podem ser vistos como fatores que afetam a estrutura familiar, quanto mais indivíduos menor a renda per capita e maior a possibilidade de aumento do número de dependentes fazendo crescer a necessidade de consumo e o trade off entre trabalhar e estudar níveis elevados de educação. Em relação às horas trabalhadas, mais horas trabalhadas representam menor tempo da pessoa de referência para serem dedicadas à família.

Dentre os fatores relacionados ao mercado de trabalho local, apenas 0 salário médio dos adultos com idade entre 26 e 65 anos não foi relevante. 0 salário médio auferido pelos jovens entre 14 e 20 anos e a taxa de ocupação são relevantes, como esperado para explicar o avanço entre ciclos escolares. Os dois fatores estão ligados ao custo de oportunidade entre trabalhar e estudar. Quanto maior o salário médio pago aos jovens e quanto maior a disponibilidade de emprego maior será o custo de estudar e, portanto, menor a sua utilidade.

Para as características de local de residência, como esperado, residir na zona rural reduz a utilidade de avanço entre ciclos escolares devido à menor incidência de escolas e universidades e, consequentemente, aos maiores custos de transação envolvidos no deslocamento até centros onde estejam disponíveis tais serviços educacionais. Com argumentos similares, porém em sentido contrário, justifica-se a maior utilidade que é atribuída a níveis mais elevados de escolaridade por indivíduos que residem em regiões metropolitanas.

Frente aos resultados empíricos, evidenciou-se que para a decisão de avanço entre ciclos escolares a não consideração do background familiar e de características geográficas e, em menor amplitude, fatores referentes ao mercado de trabalho podem enviesar a análise. Nesse sentido, a formulação de políticas públicas de universalização do acesso ao ensino e de aumento da escolaridade média da população, investimento em capital humano, devem levar em consideração aspectos paralelos que suportem o avanço entre ciclos escolares, principalmente promoção do aumento da renda.

5.2 Efeitos marginais médios: evidência da importância da habilidade inata para o avanço entre ciclos escolares

Muito embora os sinais dos coeficientes do modelo de escolhas ordenadas indiquem o sentido da relação entre as variáveis e a utilidade associada ao avanço entre ciclos escolares, sua interpretação não é muito intuitiva, sendo uma alternativa a observação dos efeitos marginais médios. A Tabela 3 apresenta os efeitos marginais médios entre os diferentes ciclos escolares.

Novamente, praticamente todos os coeficientes de efeitos marginais médios foram significantes ao nível de $1 \%$. Por esta razão omitiram-se os erros padrão dos mesmos e identificou-se entre parênteses os que não foram significativos ao nível de $1 \%$.

Como se observa, todas as variáveis possuem a mesma relação tanto em termos da variável latente quanto da probabilidade de avanço entre ciclos escolares, exceto para a probabilidade de estar cursando o ensino fundamental. Dessa forma, fatores que aumentam a utilidade também exercem impacto 
positivo sobre a probabilidade de conclusão de níveis mais elevados de educação, o contrário é válido para os fatores que reduzem a utilidade.

Tabela 3: Efeitos marginais médios sobre o avanço entre ciclos escolares, 2009

\begin{tabular}{|c|c|c|c|c|c|}
\hline \multicolumn{6}{|c|}{ Efeito marginal médio } \\
\hline Características & $\begin{array}{c}\text { Fundamental } \\
\text { Incompleto/Cursando }\end{array}$ & $\begin{array}{c}\text { Fundamental } \\
\text { Cursando/Completo }\end{array}$ & $\begin{array}{l}\text { Fundamental } \\
\text { Completo/Médio }\end{array}$ & Médio/Superior & $\begin{array}{l}\text { Superior/ } \\
\text { Pós- } \\
\text { Graduação }\end{array}$ \\
\hline \multicolumn{6}{|l|}{ Individuais } \\
\hline Idade & 0.0015 & -0.0024 & -0.0066 & -0.0011 & 0.0000 \\
\hline Branco & -0.0056 & 0.0088 & 0.0247 & 0.0040 & 0.0002 \\
\hline Masculino & 0.0090 & -0.0145 & -0.0402 & -0.0066 & -0.0003 \\
\hline Pessoa de referência & -0.0054 & 0.0080 & 0.0231 & 0.0038 & 0.0002 \\
\hline Transferência de renda & $(0.0008)$ & $(-0.0013)$ & $(-0.0036)$ & $(-0.0006)$ & $(0.0000)$ \\
\hline \multicolumn{6}{|l|}{ Familiares } \\
\hline Renda familiar per capita (In) & -0.0245 & 0.0389 & 0.1082 & 0.0177 & 0.0008 \\
\hline Número de pessoas & 0.0011 & -0.0017 & -0.0048 & -0.0008 & 0.0000 \\
\hline Escolaridade da pessoa de ref. & -0.0095 & 0.0151 & 0.0419 & 0.0068 & 0.0003 \\
\hline Estado civil da pessoa de ref. & -0.0060 & 0.0104 & 0.0276 & 0.0045 & 0.0002 \\
\hline Horas trab. pela pes. de ref. & 0.0001 & -0.0001 & -0.0004 & -0.0001 & 0.0000 \\
\hline Experiência da pes. de ref. & -0.0002 & 0.0003 & 0.0007 & 0.0001 & 0.0000 \\
\hline \multicolumn{6}{|l|}{ Mercado de trabalho } \\
\hline Salário médio dos jovens (In) & 0.0169 & -0.0268 & -0.0744 & -0.0121 & -0.0005 \\
\hline Salário médio dos adultos (In) & $(-0.0018)$ & $(0.0029)$ & $(0.0080)$ & $(0.0013)$ & $(0.0001)$ \\
\hline Taxa de ocupação & 0.0011 & -0.0017 & -0.0048 & -0.0008 & 0.0000 \\
\hline \multicolumn{6}{|l|}{ Geográficas } \\
\hline Rural & 0.0036 & -0.0066 & -0.0172 & -0.0028 & -0.0001 \\
\hline Região metropolitana & -0.0037 & 0.0055 & 0.0159 & 0.0026 & 0.0001 \\
\hline
\end{tabular}

O fato das variáveis exercerem efeito marginal oposto para a probabilidade de estar cursando o ensino fundamental corrobora com os resultados de que o background familiar assim como o mercado de trabalho e 0 local de residência exercem efeitos sobre a decisão de progredir entre ciclos escolares.

Para o efeito marginal médio de o indivíduo avançar entre os diferentes ciclos escolares, a maior influência dos fatores está ligada a conclusão do ensino médio. De todas as variáveis possuir maior renda familiar per capita, ter como referência pessoas com maior escolaridade e casadas, além do salário médio local dos jovens são as que mais afetam a probabilidade de avanço entre os ciclos escolares. 
Como visto na seção anterior, esses fatores estão diretamente ligados com a renda. Torna-se claro que as características supracitadas estão ligadas ao condicionamento de renda ou maior nível de renda como a escolaridade (BECKER, 1962; MINCER, 1974). Volta-se atenção, outra vez, para o papel desenvolvido pela renda quanto ao aumento da probabilidade de avanço entre ciclos escolares. Os resultados apontam que direta ou indiretamente a renda é o maior condicionante do aumento da escolaridade e avanço entre ciclos escolares.

Dentre os aspectos que reduzem a probabilidade de avanço entre ciclos escolares, os que possuem maior influência são raça/cor, gênero e residir na zona rural. Indivíduos que residem na zona rural possuem menor probabilidade de avanço entre ciclos escolares, devido a fatores já abordados.

Nesse sentido, políticas de mitigação de pobreza, ou seja, de acesso a renda são importantes canais de aumento da escolaridade, pois permitem a redução do trade off entre consumo e educação em níveis mais altos, além de possibilitar a solvência dos custos associados à educação como a aquisição de materiais didáticos, roupas dentre outros (BLANDEN et al, 2002). Outro ponto que também demanda esforço e investimento do poder público é a redução dos custos de transação envolvidos no deslocamento dos indivíduos que residem em áreas rurais.

Apesar das características consideradas neste estudo exercerem impacto significativo sobre a decisão de avançar entre ciclos escolares, a maior influência verificada foi de fatores relacionados de alguma forma com a renda. No entanto, os efeitos marginais considerados foram sobre a média das variáveis, ou seja, o impacto é observado em variações acima da média para variáveis contínuas e quando a decisão é alterada no caso das dicotômicas. Além disso, o efeito das variáveis reduz-se expressivamente conforme a decisão passa para ciclos mais elevados de educação.

Segundo Cameron e Heckman (1998 e 2001), esses resultados estão de acordo com o que se encontra frequentemente na literatura. Frente aos fatos, 0 avanço entre níveis elevados de educação depende de variáveis não incluídas ou não observáveis como a habilidade inata do indivíduo, o que vai de encontro a estudos como o de Griliches (1977) e Farber e Gibbons (1996), dentre outros, onde se argumenta que o nível de escolaridade, e o retorno em termos de rendimento provido pelo mesmo, seriam em última instância função dessa característica inerente aos agentes. Para verificar esse argumento com maior afinco seria necessário controlar o efeito da habilidade inata, o que foge do escopo deste artigo.

\section{Considerações finais}

O Brasil destaca-se atualmente no cenário interno e externo como forte economia emergente, situação justificada pelos indicadores de crescimento econômico e ascensão social por ele apresentados. Apesar disso, o mesmo também é destaque pela alta concentração e desigualdade na distribuição de renda. O retrato da educação no país evidencia que se precisa de maior investimento e políticas públicas na área. A escolaridade média de 7,15 anos está abaixo do ensino fundamental previsto como obrigatório, 8 anos, na Constituição Federal de 1988. 
A baixa média de escolaridade no Brasil é resultado dos $44,6 \%$ da população acima de 15 anos que não completaram ou simplesmente desistiram do ensino fundamental. Frente a esta constatação e os objetivos propostos pelo novo PNE (2011-2020), o presente artigo buscou no background familiar, mercado de trabalho e fatores geográficos a explicação para o avanço entre ciclos escolares no Brasil.

A análise empírica mostrou que a exclusão das características supracitadas causam viés de omissão de variável relevante, corroborando com a teoria e literatura de que características familiares e do mercado de trabalho são importantes na tomada de decisão quanto ao nível educacional. Fatores geográficos também se mostraram importantes, principalmente, o fato de residir na zona rural que impõe custos de transação adicionais para obter níveis mais elevados de educação.

Constatou-se que vários fatores estavam ligados direta ou indiretamente com a renda, evidenciando que esse seria um canal condicionante para o aumento da escolaridade (capital humano). Políticas públicas que foquem a mitigação da pobreza podem exercer efeitos positivos sobre a quantidade de educação no país, como evidenciado pela renda per capita.

Por fim, chegou-se a conclusão de que mesmo significativos, as características consideradas exercem pouca influência sobre a decisão de completar níveis elevados de educação, que estaria mais ligada à habilidade inata do indivíduo.

\section{REFERÊNCIAS}

ALTONJI, J. G.; BLOM, E.; MEGHIR, C. Heterogeneity in human capital investments: High school curriculum, college major, and careers. NBER Working Paper n. 17985. 2012.

BECKER, G. S. Investment in human capital: A theoretical Analisys. Journal of Political Economy. v. 70, n. 5, p. 9-49, 1962.

BLANDEN, J.; GREGG, P.; MACHIN, S. Education and family income. Disponível em: http://personal.Ise.ac.uk/machin/pdf/Education\%20and\%20Family\%20Income\% 20Stoke\%20Rochford\%20Final\%20Version.pdf. Acesso em 17 de Junho de 2013.

BRANT R. Assessing proportionality in the proportional odds model for ordinal logistic regression. Biometrics. vol. 46 n.4, p. 1171-8. DOI: 10.2307/2532457, 1990.

CÂMARA DOS DEPUTADOS. Projeto de lei do plano nacional de educação - PNE (2011-2020). Biblioteca Digital da Câmara dos Deputados. Brasília. 2011. Disponível em: http:/ /bd.camara.gov.br

CAMERON, A. C.; TRIVEDI, P. K. Microeconometrics methods and applications. Cambridge: Cambridge University Press. 1058 p. 2005. 
CAMERON, S.; J. HECKMAN. Life cycle schooling and dynamic selection bias: models and evidence for five cohorts of American males. Journal of Political Economy. v.106, p. 262-333. 1998.

CAMERON, S.; J. HECKMAN. The dynamics of educational attainment for black, hispanic and white males. Journal of Political Economy. v. 109, p. 455-99. 2001.

CARVALHO, A. P. de.; NERI, M. C.; SILVA, D. B. Diferenciais de Salários por Raça e Gênero: Aplicação dos procedimentos de Oaxaca e Heckman em Pesquisas Amostrais Complexas. XV Encontro de Estudos Populacionais, 18 a 22 setembro de 2006, ABEP. Dezembro de 2006 - Disponível em: http://bibliotecadigital.fgv.br/dspace/bitstream/handle/10438/934/2255.pdf?sequ ence $=1$

COURSEIL, C. H.; SANTOS, D. D.; FOGUEL, M. N. Decisões críticas em idades críticas: A escolha dos jovens entre estudo e trabalho no Brasil e em outros países da América Latina. Texto para Discussão n. 797. 2001.

FARBER, H. S.; GIBBONS, R. Learning and wage dynamics. The Quarterly Journal of Economics. v. 111, n. 4, p. 1007-1047. 1996.

GRILICHES, Z. Estimating the Returns to Schooling: Some Econometric Problems, Econometrica, Vol. 45, n. 1, pp. 1-22. 1977.

HANUSHEK, E. A.; LEUNG, C. K. Y.; YILMAZ, K. Redistribution through education and others transfer mechanisms. NBER Working Paper n. 8588. 2001.

HANUSHEK, E. A.; WOESSMANN, L. Schooling, cognitive skills, and the Latin American growth puzzle. NBER Working Paper n. 15066. 2009.

INSTITUTO DE PESQUISA ECONÔMICA APLICADA - IPEA. Financiamento da educação: Necessidades e possibilidades. Comunicado n. 124. 2011.

KERSTENETZKY, C. L.; ALVARENGA, L. V. B. H. Déficit de educação no Brasil: uma estimativa. Centro de Estudos sobre Desigualdade e Desenvolvimento - CEDE. Texto para Discussão n. 16. 15 p. 2009.

LEON, F. L. L.; MENEZES-FILHO, N. A. Reprovação, avanço e evasão escolar no Brasil. Pesquisa e Planejamento Econômico. v.32, n.3, p. 417-452. 2002.

LEVIN, H. M. Mapping the economics of education. An introductory essay. Educational researcher. vol. 18, n. 4, p. 13-16, 1989.

LONG, J. S.; FREESE, J. Regression Models for Categorical Dependent Variables Using Stata. 2. ed. Stata Press, 2006.

MINCER, J. Schooling, experience, and earnings. National Bureau of Economic Research: Columbia University Press, New York, 1974.

OAXACA, R. Male-Female Wage Differentials in Urban Labor Market. In International Economic Review. v. 14, n.3, p. 693-709. 1973 - Disponível em: 


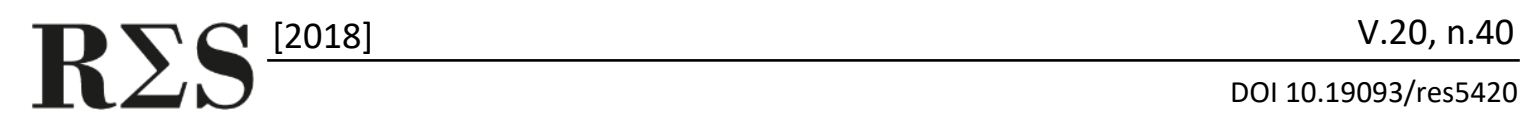

http://wwwbcf.usc.edu/ ridder/Lnotes/Undeconometrics/Transparanten/Wagede comp.pdf

PEGORINI, L. P. CASTRO, J. L. S. LIMA, J. E. Rendimento dos jovens no mercado de trabalho: uma análise para a Região Centro Oeste. Anais do $\mathbf{X}$ Encontro Nacional da Associação Brasileira de Estudos Regionais. RecifePE, 2012.

SANTOS, G. C.; FONTES, R. M. O.; BASTOS, P. M. A.; LIMA, J. E. Mercado de trabalho e rendimento no meio rural Brasileiro. Economia Aplicada. v. 14, n. 3, p. 355-379, 2010.

SILVA, P. L. N.; PESSOA, D. G. C.; LILA, M. F. Análise estatística de dados da pnad: incorporando a estrutura do plano amostral. Ciência \& Saúde Coletiva. v.7, p. 659-670. (2002).

SOUZA, A. P.; PONCZEK, V.; OLIVA, B. Os determinantes do fluxo escolar entre o ensino fundamental e o ensino médio no Brasil. Texto para Discussão 286 FGV. 2011.

WOLFE, R.; W. GOULD. An approximate likelihood-ratio test for ordinal response models. Stata Technical Bulletin. StataCorp LP, vol. 7 n.42. 1998. 